The Egyptian Journal of Hospital Medicine (January 2021) Vol. 82 (1), Page 11-20

\title{
The Role of Functional MRI in The Evaluation of The Treated Head and Neck Squamous Cell Carcinoma
}

\author{
Ahmed S. Abdelrahman*, Amgad S. Abdel-Rahman, Mena E.Y. Ekladious \\ Radiology Department - Faculty of Medicine, Ain Shams University, Cairo, Egypt \\ *Corresponding author: Ahmed S. Abdelrahman; Mobile: 01200220999; Email: dr_ahmedsamy@yahoo.com
}

\begin{abstract}
Background: The diffusion-weighted imaging (DWI) with apparent diffusion coefficient (ADC) map and the dynamic contrast-enhanced magnetic resonance imaging (DCE-MRI) constitute the functional MRI technique with many oncological applications as it can define tissue microstructure and characterize the post-treatment changes from a recurrent tumor in treated head and neck squamous cell carcinoma (HNSCC).

Objective: To evaluate the role of the DWI, ADC map and DCE-MRI for characterization of post-treatment changes and recurrent tumors in patients with treated HNSCC.

Patients and Methods: Forty-five patients with treated HNSCC were included in this study with mean ages of $59 \pm$ 7.5 years. The patients underwent pre and post-contrast head and neck MRI, DWI with ADC map and DCE-MRI, each study diagnosis was then compared to the patient outcome.

Results: The sensitivity and specificity of the qualitative DWI (DW-MRI) (87.5\% and $82.8 \%$ respectively) were higher than the conventional MRI (75\% and 75.9\% respectively). The ADC cut-off point for the diagnosis of HNSCC recurrence was $1.095 \times 10^{-3} \mathrm{~mm}^{2} / \mathrm{s}$ with sensitivity and specificity of $87.5 \%, 86.2 \%$ respectively. The DCEMRI revealed a significant difference in the type of time intensity curve (TIC) between post-treatment changes and recurrent lesion ( $\mathrm{p}$-value $<0.0001$ ).

Conclusion: The DWI, ADC and the DCE-MRI can be used as a synchronized tool for the differentiation between post-treatment changes and recurrent tumor in treated HNSCC patients.
\end{abstract}

Keywords: HNSCC, MRI, DWI, ADC, DCE-MRI.

\section{INTRODUCTION}

Head and neck squamous cell carcinoma (HNSCC) is a major medical cause of morbidity and mortality. The incidence of tumor recurrence after treatment was $15 \%-50 \%{ }^{(1-3)}$.

Magnetic resonance imaging (MRI) can accurately delineate the tumor spread and it is considered the investigation of choice for the evaluation of head and neck cancer. However, it exhibited limited results after surgery or radiation therapy ${ }^{(4)}$. The surgical treatment distorts the normal anatomical structure. It is associated with edema and fibrosis especially after radiotherapy. This renders accurate differentiation between tumor recurrence and the post-treatment changes by the conventional MRI technique to be difficult ${ }^{(5)}$. Although the recurrent tumor demonstrated higher signal intensity in T2weighted images (WI) than the benign fibrotic change exhibited, yet further studies have demonstrated that the non-neoplastic inflammation and edema may also demonstrate hyperintense signal in T2 $\mathrm{WI}^{\left({ }^{(5)}\right.}$.

The functional MRI study included the diffusion-weighted imaging (DWI) and the dynamic contrast-enhanced magnetic resonance imaging (DCEMRI), both can delineate the microstructure of different tissues, which provide a new approach for imaging the neoplastic lesions ${ }^{(5)}$. DCE-MRI can characterize the physiological properties of the lesion including the lesion hypoxia and the angiogenesis ${ }^{(5)}$. It was validated as a useful technique for differentiating the benign from the malignant lesions and for discrimination between tumor recurrence and the posttreatment changes in the breast and pelvic masses.

DWI with apparent diffusion coefficient (ADC) map measures the differences in water mobility at different tissue microstructures ${ }^{(6)}$. The more cells number and nucleus dimensions in the malignant lesions lead to limited water molecules diffusion and, consequently to lower $\mathrm{ADC}$ values noted in the malignant lesion. On the other hand, decreased cellularity and the presence of edema and inflammatory changes in the benign and post-treatment changes lead to higher ADC values of the necrotic, edematous and fibrotic changes ${ }^{(7)}$.

The aim of this study was to delineate the value of the functional MRI including both the DWI, ADC map and DCE-MRI for differentiating the residual/recurrent tumors and the post-treatment changes in the treated HNSCC.

\section{PATIENTS AND METHODS}

This retrospective study was performed in our institution hospital during the period from February 2018 to September 2020. it included 45 patients with 
https://ejhm.journals.ekb.eg/

treated HNSCC: 28 males (62.2\%) and 17 females $(37.8 \%)$ with mean ages of $59 \pm 7.5$ years (range 44 to 73 years).

Inclusion criteria: Patients with treated HNSCC whom post-treatment imaging surveillance was performed by the MRI, DWI and DCE-MRI with no sex predilection.

Exclusion criteria: Included patients with head and neck cancer other than squamous cell carcinoma, MRI study missing DWI or DCE-MRI, and patients with absent outcome data.

Tumor recurrence was reported in cases of positive tissue biopsy, verification of clinical recurrence by the clinical examination, or progression of the disease in follow-up studies according to per Response Evaluation Criteria in Solid Tumors. Negative tumor recurrence was documented when the follow-up scan showed no abnormal features with associated negative clinical recurrence or negative tissue biopsy.

\section{Ethical approval:}

This study was approved by the Ethical Committee of Ain Shams University (FWA 000017585); Reference Number of approval: R47/2020. The requirement of written consent was waived for this retrospective study. A signed written consent was obtained from each patient.

\section{Technique of the MRI examination}

MR images were performed on a $1.5 \mathrm{~T}$ system (Achieva 1.5T, Philips Medical Systems) using head and neck coil. All patients underwent DWI and DCEMRI examinations in addition to the conventional MRI.

The conventional MRI study consisted of MR imaging covering the entire neck extending from the skull base down to the root of the neck. Sequence: axial T2WIs (TE: $10 \mathrm{~ms}$, TR: $7039 \mathrm{~ms}$ ), axial T1WIs (TE: $21 \mathrm{~ms}$, TR: $633 \mathrm{~ms}$ ), sagittal T2WI (TE: $100 \mathrm{ms,}$ TR: $3196.7 \mathrm{~ms}$ ), coronal T1 (TE:14 ms, TR: $555 \mathrm{~ms}$ ) and coronal T2 STIR (TR: $3500 \mathrm{~ms}$, TI: $150 \mathrm{~ms}$, TE: $80 \mathrm{~ms}$ ). The slice thickness was $4 \mathrm{~mm}$.

DWI was performed by using a single-shot spin-echo EPI sequence with the following parameters: axial plane 4-mm section thickness, the field of view was $240 \mathrm{~mm}$, and b-values was 0 and $1000 \mathrm{~s} / \mathrm{mm}^{2}$.

The DCE-MRI was performed using a threedimensional spoiled gradient recalled echo (3D-SPGR) sequence (TR: $5 \mathrm{~ms}$, TE: $1.4 \mathrm{~ms}$, flip angle: $15^{0}$, section thickness: $4 \mathrm{~mm}$, the field of view: $240 \mathrm{~mm}$ ). Gadolinium (Meglumine Gadopentetate, Magnevist) was injected intravenously at a dose of $0.1 \mathrm{ml} / \mathrm{kg}$ body weight with an injection rate of $2 \mathrm{ml} / \mathrm{s}$, followed by a $20 \mathrm{ml}$ saline flush. In each patient, 5 scans were obtained sequentially (around $38 \mathrm{~s}$ for each scan with a total of about $6.3 \mathrm{~min}$

After the dynamic scan a post-contrast T1WI with fat saturation was obtained in the axial (TR: 611ms, TE: $21 \mathrm{~ms}$ ), coronal (TR: 570, TE: $14 \mathrm{~ms}$ ), and sagittal (TR: $570 \mathrm{~ms}$, TE: $14 \mathrm{~ms}$ ) planes.

\section{Imaging Evaluation}

The conventional MRI examination was independently evaluated by two head and neck radiologists (with 11 and 9 years of experience), and then each radiologist was asked to reevaluate the MRI examination combined with DWI and ADC map. Three months later, the time intensity curve (TIC) was evaluated while the radiologists were blinded to the conventional MRI images and the DWI data. The disagreement was resolved and managed by consensus.

The evaluation of the conventional MRI was performed according to the following features: tumor recurrence was reported if there was mass-like lesion with intermediate T2 WI signal and moderate postcontrast enhancement. Post-treatment fibrosis was diagnosed if a linear or triangular lesion is noted exhibiting very low T2 WI signal and low T1 WI signal with absent or faint post-contrast enhancement. Post-treatment inflammation and edema were recognized if there was diffuse lesion exhibiting high signal in T2 WI and low signal intensity in T1 WI with strong enhancement in the post-contrast study ${ }^{(8,9)}$.

Tumor recurrence in the DWI (qualitative DWI) was diagnosed if the lesion exhibited high or very high signal in the DWI with $b$ value of 1000 $\mathrm{s} / \mathrm{mm}^{2}$ associated with low signal in the ADC map, yet the post-treatment change was diagnosed if these features were missed. Quantitative ADC value was measured by placing a region of interest (ROI) in the solid portion and the most hypointense area of ADC map. Three ADC values were calculated, and the mean ADC value was used in the statistical analysis ${ }^{(10)}$.

The DCE-MRI images were transferred to Philips IntelliSpace Portal Image and information management software V 9.0. (Philips Medical System Nederland B.V), where ROI was manually drawn over the most enhancing area with avoiding the necrotic lesion, the time intensity curve was then generated automatically. Three TIC types were qualitatively recognized: continuous rising curve (type 1), rapid uptake with gradual washout or plateau curve (type 2) and rapid uptake with rapid washout curve (type 3) (Fig.1). 

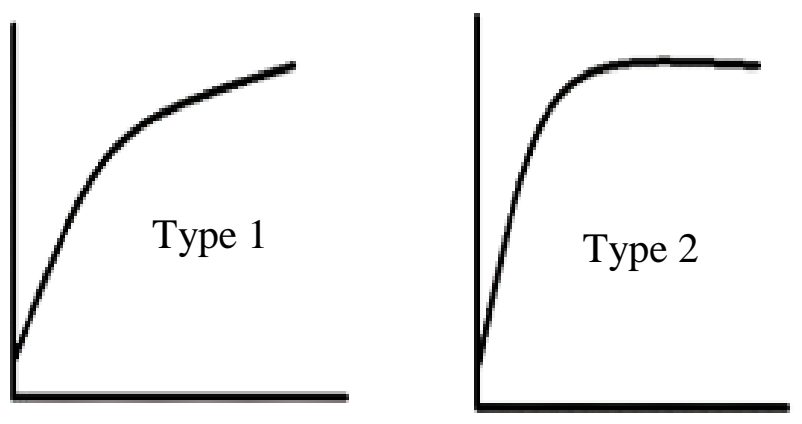

Figure (1): Different types of the time intensity curve (TIC)

\section{Statistical analysis}

The data were analyzed using Statistical Package for Social Science (IBM Corp, released 2013. IBM SPSS statistics for windows, V. 22.0. Armonk, NY. USA). Parametric quantitative data were expressed as mean \pm standard deviation (SD). Qualitative data were described as frequency and percentage. The categorical variable was analyzed with Pearson Chi-Square test and Fisher's Exact Test. The cross-tabulation was used to evaluate the diagnostic performance, sensitivity, specificity, and accuracy. Analysis of Receiver operator characteristic (ROC) was performed to determine the optimal cut off value. P-value $\leq 0.05$ was considered statistically significant.

\section{RESULTS}

This study included 45 patients with treated HNSCC (29 laryngeal, 6 oral cavity, 4 nasopharyngeal, 3 oropharyngeal, 2 sinonasal and 1 hypopharyngeal squamous cell carcinoma lesions) presenting to our institution for routine surveillance with a mean age of
$59 \pm 7.5$ years (44 to 73 years). The study included 28 $(62.2 \%)$ males and $17(37.8 \%)$ females.

The final diagnosis revealed recurrent/residual tumor lesion in 16 patients and post-treatment changes in 29 patients. Yet the conventional MRI which included pre-contrast and post-contrast non-dynamic sequence revealed recurrent lesions in 19 patients (12 were true positive and 7 were false positive) and posttreatment changes in 26 patients ( 22 were true negative cases and 4 were false-negative cases), with sensitivity, specificity, and accuracy of $75 \%, 75.9 \%$ and $75.5 \%$ respectively (Tables $1 \& 2$ ).

The conventional MRI examination combined with qualitative DWI (DW-MRI) revealed recurrent tumor with true diffusion restriction noted inside the lesion in 19 patients (14 were true positive cases and 5 were false-positive cases). Post-treatment changes in 26 patients (24 were true negative and 2 were false negative). The diagnostic performance of qualitative DW-MRI was higher with sensitivity, specificity, and accuracy of $87.5 \%, 82.8 \%$ and $84.4 \%$ respectively (Table $1 \& 2$ ) \& (Fig. 2, $3 \& 4$ ).

Table (1): Distribution of positive and negative cases diagnosed by the conventional MRI, MRI with qualitative DWI and quantitative ADC value

\begin{tabular}{|lcccc|}
\hline & TP & FP & TN & FN \\
\hline Conventional MRI & 12 & 7 & 22 & 4 \\
Conventional MRI with DWI & 14 & 5 & 24 & 2 \\
ADC less than 1.095 & 14 & 4 & 25 & 2 \\
\hline
\end{tabular}

Table (2): Diagnostic performance of the conventional MRI, MRI with qualitative DWI and quantitative ADC value

\begin{tabular}{|lccccc|}
\hline & Sensitivity & Specificity & PPV & NPV & Accuracy \\
\hline Conventional MRI & $75 \%$ & $75.9 \%$ & $63.2 \%$ & $84.6 \%$ & $75.5 \%$ \\
Conventional MRI with DWI & $87.5 \%$ & $82.8 \%$ & $73.7 \%$ & $92.3 \%$ & $84.4 \%$ \\
ADC less than 1.095 & $87.5 \%$ & $86.2 \%$ & $77.8 \%$ & $82.6 \%$ & $86.7 \%$ \\
\hline
\end{tabular}



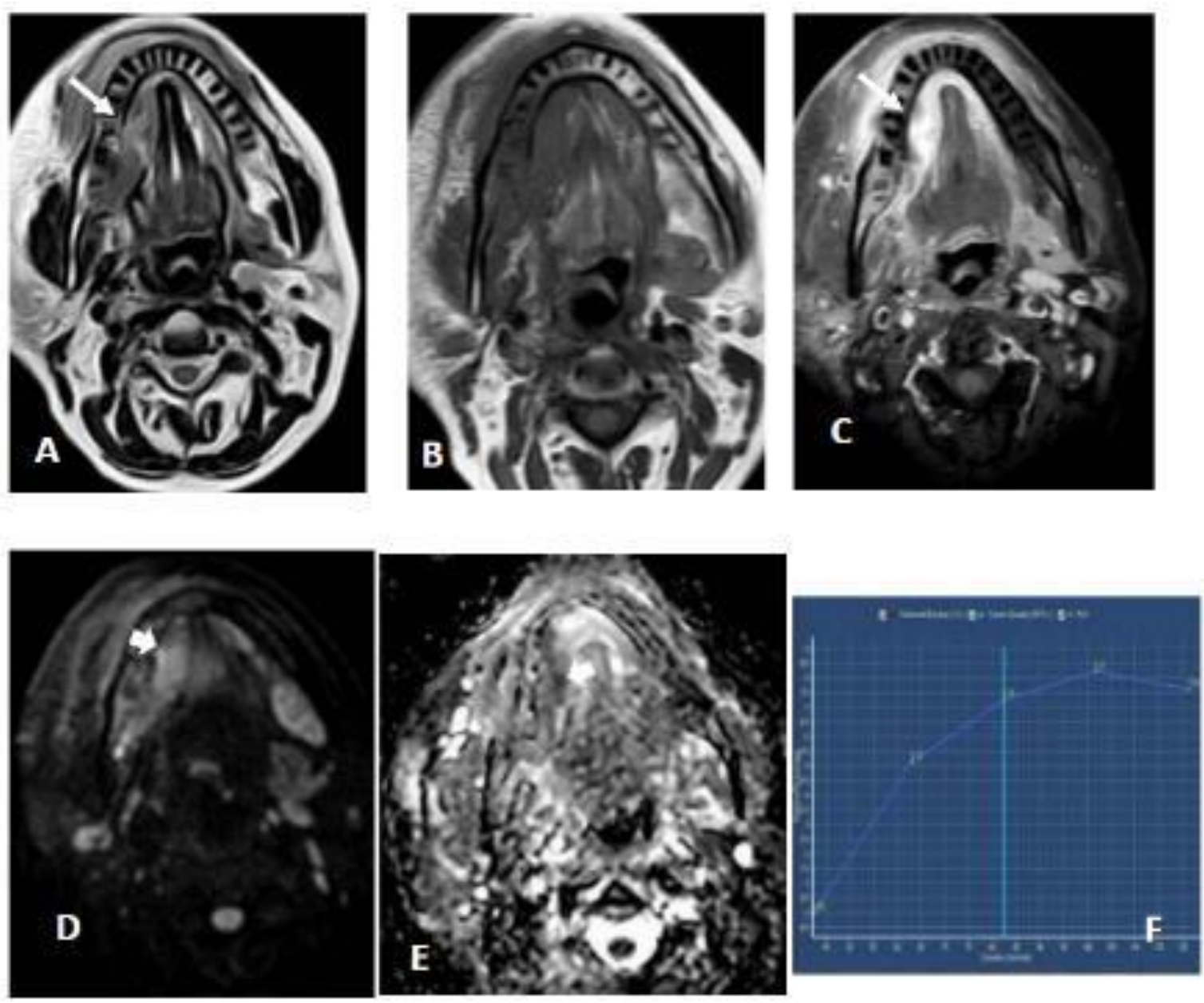

Fig (2): Known case of tongue carcinoma underwent surgery followed by radiotherapy. Axial T2 (A) shows a right hemi tongue soft tissue thickening at operative bed with high signal (arrow). Axial T1 pre (B) and post contrast (C) shows an enhancing lesion at operative bed (arrow). DWI (D) and ADC map (E) show evidence of diffusion restriction with high signal in DWI and low signal in ADC map (arrowhead), the ADC value $=0.9 \times 10^{-3} \mathrm{~mm}^{2} / \mathrm{s}$. TIC (F) shows type 3 curve with rising followed by wash out. Pathology revealed recurrent neoplasm.

The mean ADC value of the recurrent tumors $\left(0.956 \pm 0.27 \times 10^{-3} \mathrm{~mm}^{2} / \mathrm{s}\right)$ was significantly lower than that of the post-treatment changes $\left(1.58 \pm 0.29 \times 10^{-3} \mathrm{~mm}^{2} / \mathrm{s}\right)(\mathrm{p}$-value $<0.000)$ (Fig. $\left.5 \& 6\right)$. The ADC range of the recurrent tumor was $\left(0.63-1.59 \times 10^{-3} \mathrm{~mm}^{2} / \mathrm{s}\right)$ and it was $\left(0.95-2.15 \times 10^{-3} \mathrm{~mm}^{2} / \mathrm{s}\right)$ for post-treatment change. The analysis of the receiver operating curve revealed that the optimal $\mathrm{ADC}$ cut-off value for discrimination between recurrent tumor and post treatment changes was $1.095 \times 10^{-3} \mathrm{~mm}^{2} / \mathrm{s}(\mathrm{AUC}=0.931,95 \% \mathrm{CI}, 0.855$ to 1 , p-value <0.0001) (Fig. 7). At this value, 18 patients were diagnosed with tumor recurrence (14 were true positive and 4 were false positive) and 27 patients were diagnosed with post treatment changes ( 25 were true negative and 2 were false negative), with sensitivity, specificity and accuracy of $87.5 \%, 86.2 \%$, and $86.7 \%$ respectively (Tables $1 \& 2$ ). 

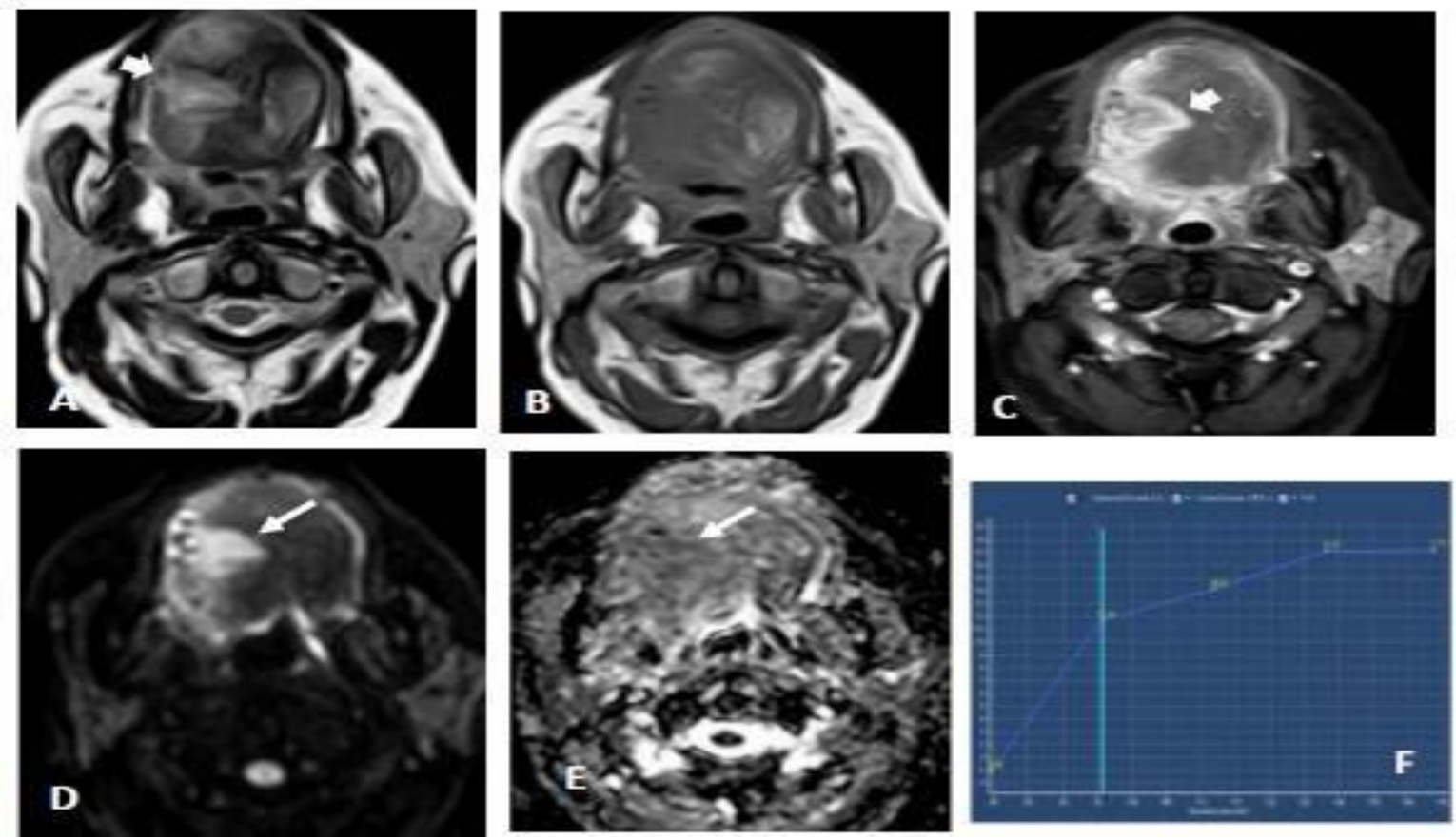

Fig (3): Known case of tongue carcinoma underwent surgery followed by radiotherapy. Axial T2 (A) shows a right hemi tongue mass lesion at operative bed with high signal (arrowhead). Axial T1 pre (B) and post contrast (C) shows heterogeneous predominantly peripheral enhancement (arrowhead). DWI (D) and ADC map (E) show evidence of diffusion restriction with high signal in DWI, the ADC shows low signal at lesion periphery (arrow), the ADC value $=0.99 \times 10^{-3} \mathrm{~mm}^{2} / \mathrm{s}$. TIC $(\mathrm{F})$ shows type 2 curve (plateau curve). Pathology revealed recurrent neoplasm.
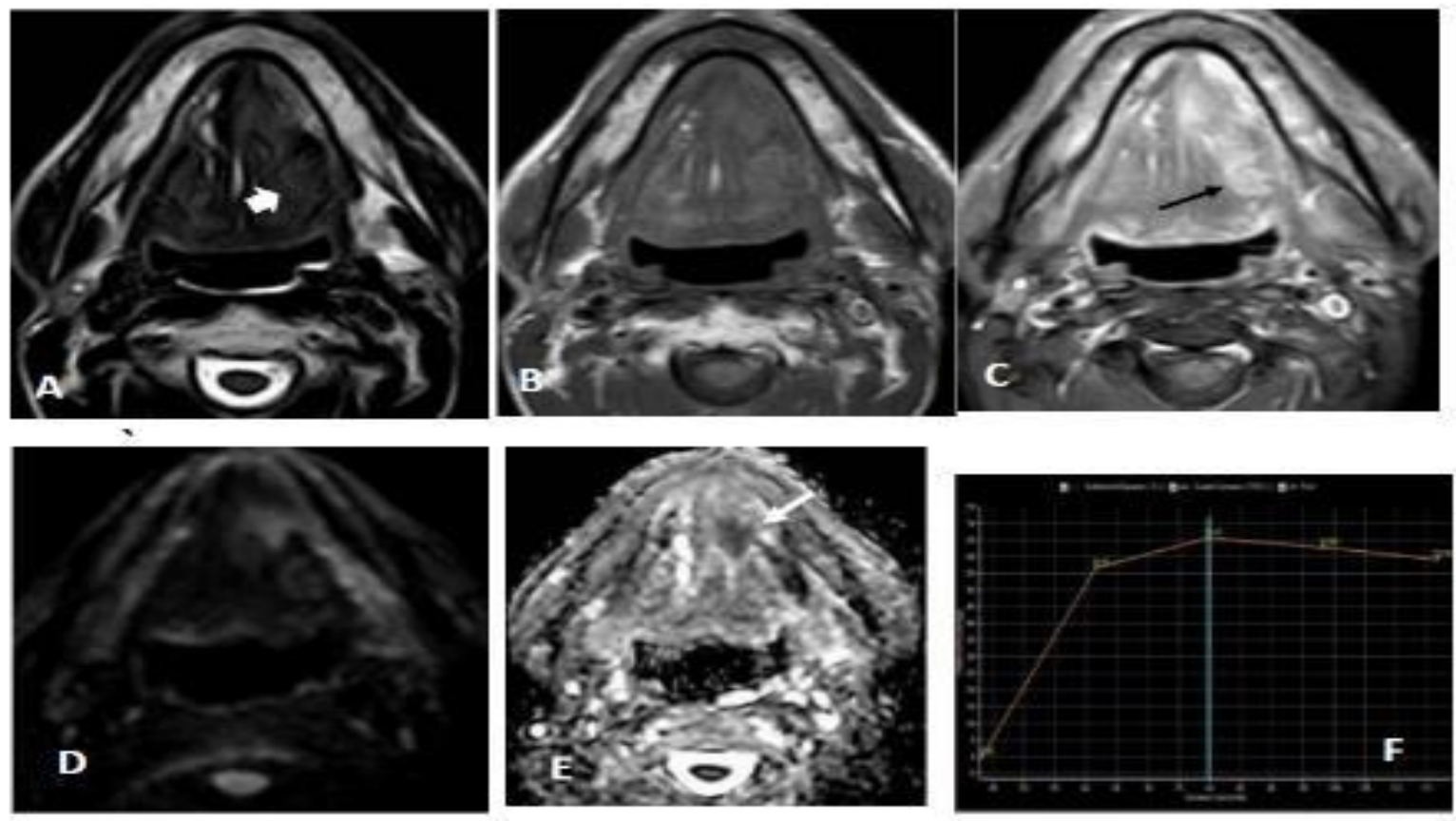

Fig (4): Known case of tongue carcinoma underwent surgery followed by radiotherapy. Axial T2 (A) shows a left hemi ill-defined lesion at operative bed with intermediate signal (arrowhead). Axial T1 pre (B) and post contrast (C) shows an enhancing lesion (black arrow). DWI (D) and ADC map (E) show evidence of diffusion restriction with high signal in DWI and low signal in ADC map (white arrow), the ADC value $=0.7 \times 10^{-3} \mathrm{~mm}^{2} / \mathrm{s}$. TIC (F) shows type 3 curve. Pathology revealed recurrent neoplasm. 
The analysis of the dynamic MRI revealed that type 3 curve was seen in $7 / 16(43.75 \%)$ of patients with recurrent lesion and type 2 curve was seen in $9 / 16$ $(56.25 \%)$. Yet type 1 curve was not detected in a patient with a recurrent tumor (Fig. 2-6). On the other hand, type 1 curve was the predominant curve in the post-treatment changes as it was detected in 25/29 $(86.2 \%)$ patients. Yet type 2 curve was seen in $4 / 29$ (13.8\%) patients. Type 3 curve was not detected in a patient with post-treatment changes (Table 3). The Fisher's Exact Test revealed a significant difference in the number of curve type among patients with recurrent lesion and those with post-treatment changes ( $\mathrm{p}$-value $<0.000)$, and there was a significant trend for tumor recurrence as the TIC increased from 1 to 3 (p-value < 0.000 ). The analysis of TIC, if type 1 curve was considered criteria for post-treatment changes, yet type 2 and 3 curves were considered criteria for tumor recurrence that revealed sensitivity, specificity, and accuracy of $100 \%, 86.2 \%$ and $91.1 \%$ respectively $(\mathrm{AUC}=0.931,95 \% \mathrm{CI}, 0.853$ to $1, \mathrm{p}$-value $<0.0001$ ) (Fig. 7). Type 2 curve was noted in four false-positive cases with negative recurrence final diagnosis.

Table (3): Dynamic MRI: Distribution of tumor recurrence and post-treatment changes among different type of TIC

\begin{tabular}{|lccccc|}
\hline & Type 1 & Type 2 & Type 3 & $\mathbf{X}^{\mathbf{2}}$ & P value \\
Tumor recurrence (\%) & $0 / 16$ & $9 / 16(56.25 \%)$ & $7 / 16(43.75 \%)$ & 36.594 & $<0.000$ \\
Post treatment changes (\%) & $25 / 29(86.2 \%)$ & $4 / 29(13.8 \%)$ & $0 / 29$ & & \\
\hline
\end{tabular}

$\mathrm{X}^{2}$ : Fisher's Exact Test
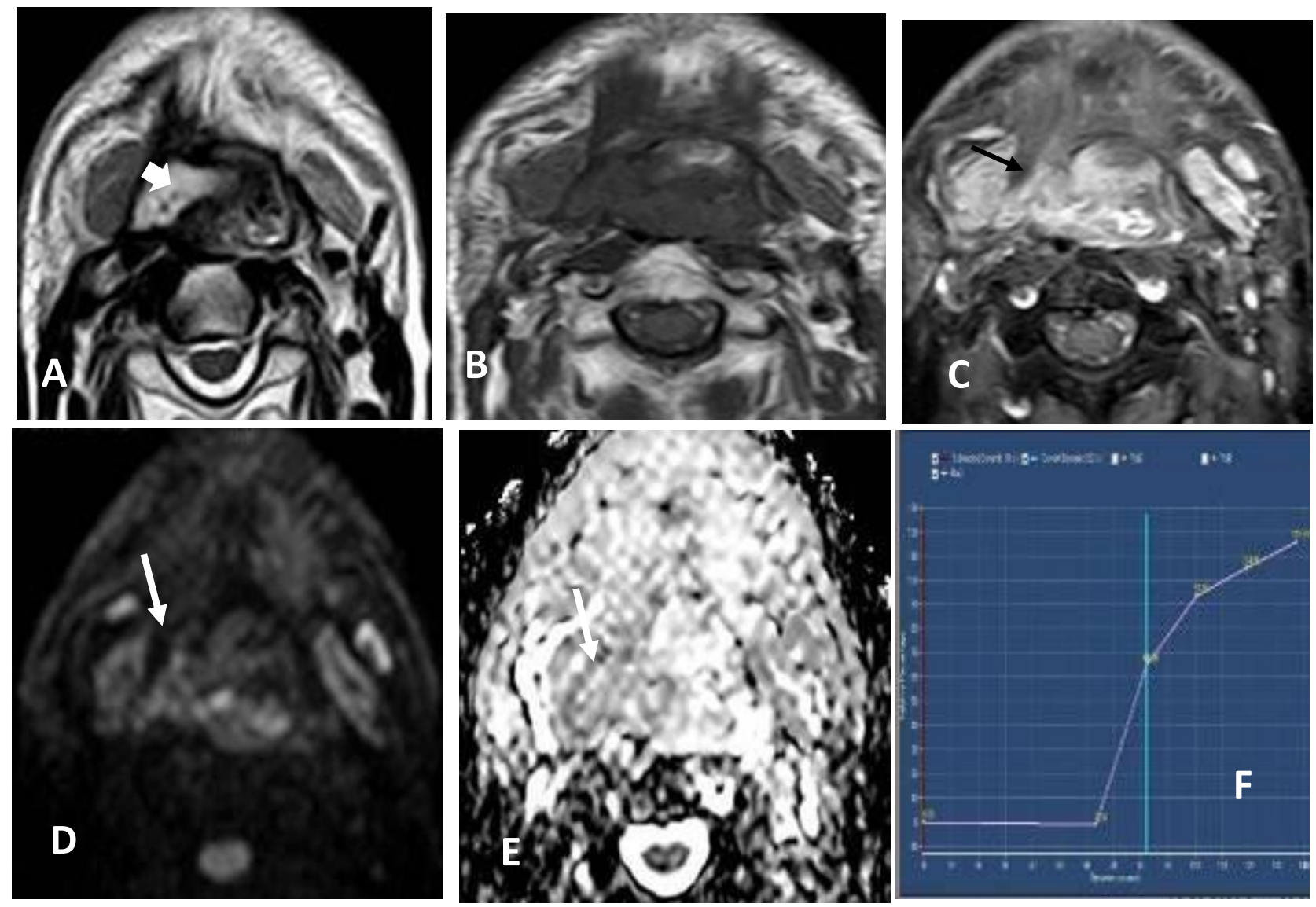

Fig (5): Known case of laryngeal carcinoma underwent total laryngectomy followed by radiotherapy. Axial T2 (A) shows a well-defined lesion is seen anterior to the neo-pharynx (arrowhead). Axial T1 pre (B) and post contrast (C) shows a lesion with heterogeneous enhancement (black arrow). DWI (D) and ADC map (E) show no evidence of diffusion restriction with low signal in DWI (white arrow), the ADC value $=1.6 \times 10^{-3} \mathrm{~mm}^{2} / \mathrm{s}$. TIC (F) shows type 1 rising curve. Pathology showed post radiation changes with no recurrent neoplasm. 

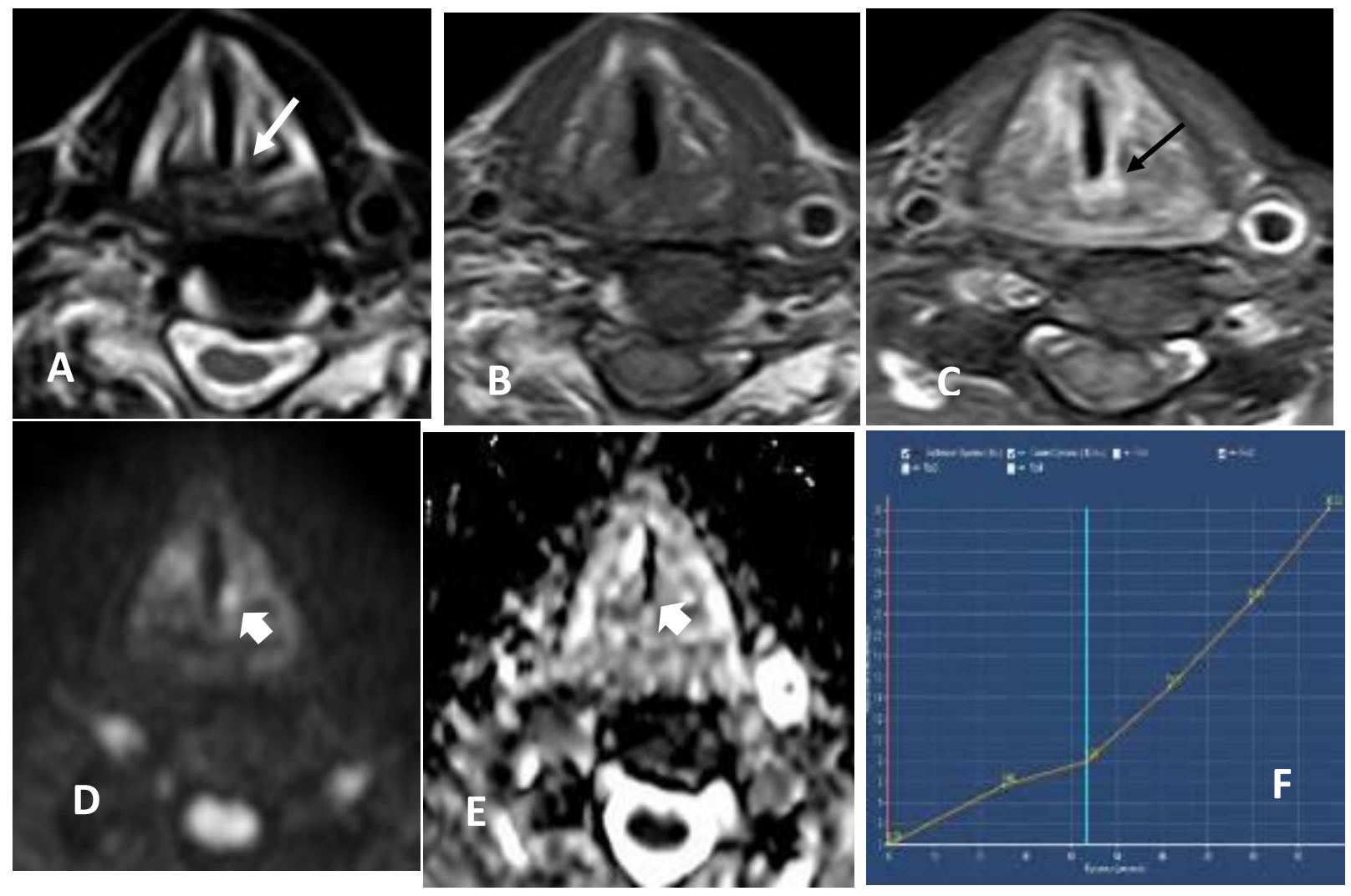

Fig (6): Known case of laryngeal carcinoma un on radiotherapy. Axial T2 (A) shows a subtle left vocal cord thickening (white arrow). Axial T1 pre (B) and post contrast (C) show subtle enhancement (black arrow). DWI (D) high signal focus in DWI with no matched low signal in ADC map (E) (arrowhead), the ADC value $=1.2 \times 10^{-}$ ${ }^{3} \mathrm{~mm}^{2} / \mathrm{s}$. TIC (F) shows type 1 rising curve. Pathology showed post radiation changes.
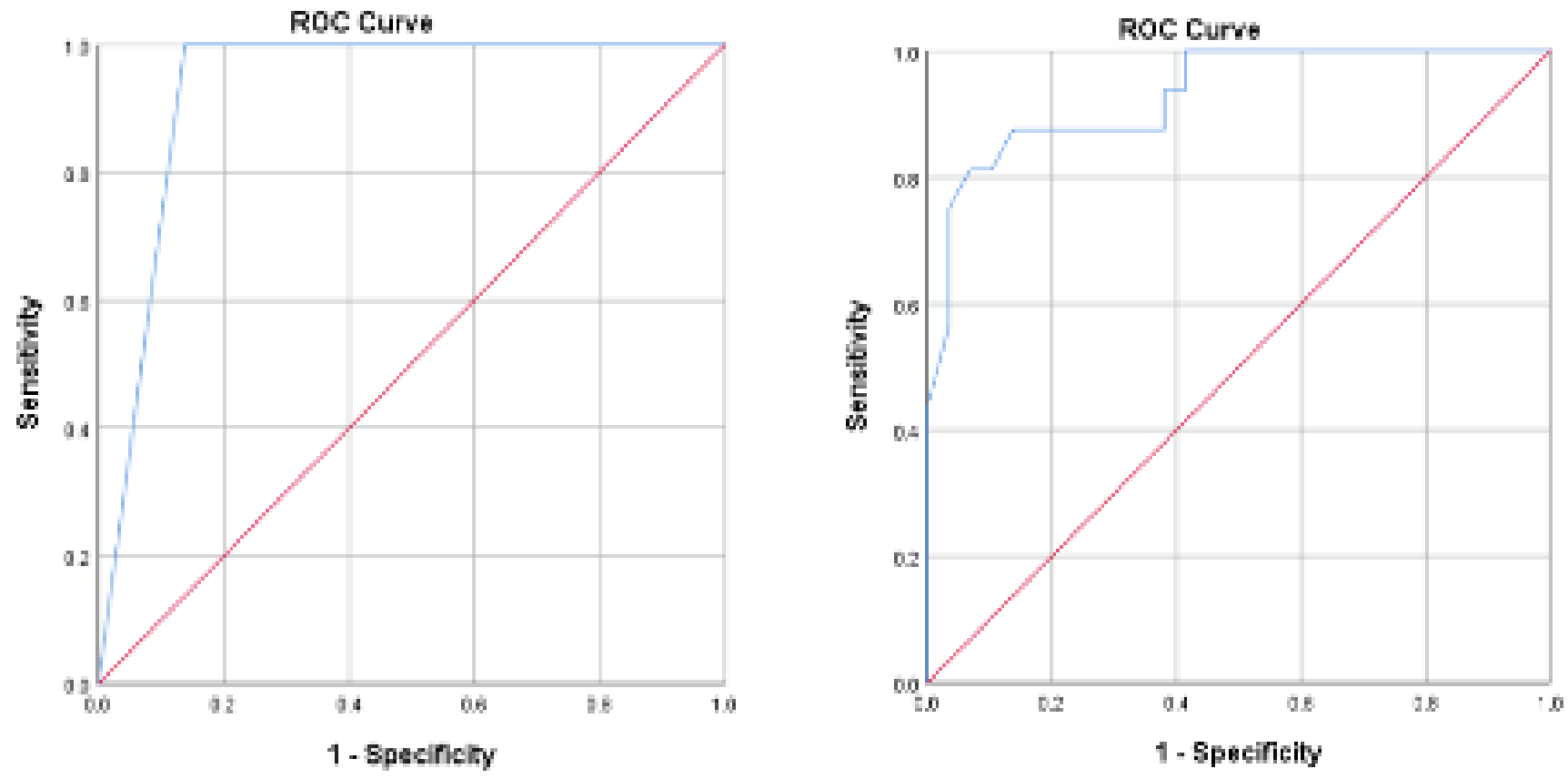

Fig. (7): ROC curve analysis of ADC value (left) and TIC (right). 
https://ejhm.journals.ekb.eg/

\section{DISCUSSION}

The post-surgical alteration of the normal anatomy as well as the edema and fibrosis secondary to radio and chemotherapy hinder proper interpretation of the conventional MRI images and render the differentiation between local recurrence and the posttreatment changes a difficult issue. As the posttreatment changes can exhibit high signal in T2 WI with contrast enhancement in the post-contrast images, which mimics tumor recurrence ${ }^{(11)}$, hence, the functional imaging technique including diffusionweighted images and the dynamic post-contrast MRI can add an important role in resolving this issue ${ }^{(5)}$. The dynamic post-contrast MRI with TIC allows the depiction of the physiologic properties of lesions. Local recurrence is characterized by hypervascularity and leaky vessels while post-treatment changes are characterized by the presence of fibrosis, which shows persistent delayed enhancement. This difference can be clearly illustrated via the dynamic MRI studies ${ }^{(\mathbf{1 2})}$. The DWI reflects the random motion of water protons, which is affected by the microstructure of tissue. Recurrent masses show high cellularity thus it shows restricted diffusion with low ADC values while posttreatment edema and fibrosis are characterized by low cellularity, which shows facilitated diffusion and high ADC values ${ }^{(13)}$.

In the current study, the diagnostic performance of the qualitative DW-MRI for diagnosis of tumor recurrence was superior to the conventional MRI as the sensitivity, specificity and accuracy of DW-MRI were $87.5 \%, 82.8 \%$ and $84.4 \%$ respectively versus $75 \%, 75.9 \%$ and $75.5 \%$ respectively in the conventional MRI. Similar results ae also concluded by Ailianou et al. (14), who revealed the superior diagnostic performance of the DW-MRI with sensitivity and specificity of $89.4 \%$ and $90.8 \%$ compared to $78.9 \%$ and $80 \%$ in the conventional MRI.

The DWI quantitative assessment using the ADC map showed that the mean ADC value of posttreatment changes was significantly higher than that of tumor recurrence $\left(1.58 \pm 0.29 \times 10^{-3} \mathrm{~mm}^{2} / \mathrm{s}\right.$ versus $0.956 \pm 0.27 \times 10^{-3} \mathrm{~mm}^{2} / \mathrm{s}$ respectively). This is consistent with previous literature data, which revealed that the mean ADC value of tumor recurrence (range 0.91 to $1.17 \times 10^{-3} \mathrm{~mm}^{2} / \mathrm{s}$ ) was lower than that of posttreatment changes (range 1.39 to $2.07 \times 10^{-3} \mathrm{~mm}^{2} / \mathrm{s}$ ) ${ }^{(15-}$ 18). The qualitative and quantitative DWI and ADC map have a beneficial diagnostic role for evaluation of post-treatment suspicious lesion helping in the characterization of tumor recurrence from the posttreatment changes.
In the current study, the optimal cut off value for characterization of tumor recurrence was $1.095 \times 10^{-}$ ${ }^{3} \mathrm{~mm}^{2} / \mathrm{s}$, with sensitivity, specificity, and accuracy of $87.5 \%, 86.2 \%$ and $86.7 \%$ respectively. At this cut off value, four post-treatment changes cases were diagnosed as false-positive cases of tumor recurrence. The DWI can still exhibit high signal at b $1000 \mathrm{~s} / \mathrm{mm}^{2}$ in the benign post-treatment fibrous and edematous tissue with consequent confusion with the restricted diffusion noted in the recurrent tumor ${ }^{(7)}$. There were also two cases with tumor recurrence, which were diagnosed as false-negative cases of post-treatment changes. The presence of edematous changes with or without post-radiation liquefactive necrosis may relatively result in higher ADC values leading to a false interpretation of post-treatment changes ${ }^{(19)}$. An overlap was noted in the range of ADC value of the recurrent tumor and the post-treatment changes (0.63$1.59 \times 10^{-3} \mathrm{~mm}^{2} / \mathrm{s}$ versus $0.95-2.15 \times 10^{-3} \mathrm{~mm}^{2} / \mathrm{s}$ respectively), which was also noted in the previously published data ${ }^{(\mathbf{1 4}-18)}$, explaining the false positive and false negative cases in multiple studies.

Other studies also reported that the DWI is an accurate study for distinguishing between recurrent/residual tumors and the radiation-induced changes with ADC cut off value range from 0.97-1.46

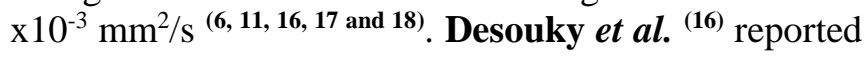
$100 \%$ sensitivity with lower ADC cut off value of $0.97 \times 10^{-3} \mathrm{~mm}^{2} / \mathrm{s}$. King et al. ${ }^{(11)}$ revealed specificity of $100 \%$ with higher cut off value of $1.4 \times 10^{-3} \mathrm{~mm}^{2} / \mathrm{s}$. The difference in the cut off value can be explained by the different parameters of diffusion-weighted images acquisition as well as the difference in histopathological criteria of tumors in the studied patients. Different cut off value revealed also different sensitivity and specificity for tumor recurrence.

The dynamic post-contrast enhancement images were evaluated qualitatively using the shape of TIC, which significantly differentiated between the post-treatment changes and the recurrent tumor. Continuous rising curve (Type 1) was only depicted in the post-treatment changes, yet type 3 curve with rapid enhancement and washout was only noted in the recurrent tumor. Type 2 curve was also found predominantly in the recurrent tumor. Previous studies also reported similar results ${ }^{(5,16,20)}$, which emphasize the value of DCE-MRI for differentiating between tumor recurrence and the posttreatment changes. A recurrent tumor is hypervascular and shows intense and early enhancement, which also is explained by the leaky vessels and high cellularity. On the other hands, the posttreatment changes and fibrosis shows delayed 
persistent enhancement due to retention of much contrast in the extravascular extracellular space ${ }^{(\mathbf{1 2})}$.

The sensitivity and specificity of DCE-MRI considering type 1 curve indicative of post-treatment changes and type 2 and 3 curves indicative of tumor recurrence were $100 \%$ and $86.2 \%$ respectively. A similar result is also noted by Choi et al. ${ }^{(31)}$, which revealed that the sensitivity and specificity of type 2 and 3 curves for tumor recurrence were $100 \%$ and $83.3 \%$ respectively. The DCE-MRI was also used to differentiate recurrence from post-treatment change in other tissue as colon, breast, and gynaecological lesion where they revealed similar results with early enhancement noted in the recurrent tumor, and rising enhancement noted in the fibrotic and scarring posttreatment changes ${ }^{(\mathbf{2 1})}$.

Our study had some limitations, first, the current study included head and neck cancers with various head and neck subsites, treatment modalities, and time intervals between last treatment and the imaging. Although, the prognosis is related to tumor location, yet the ADC value is unlikely to be significantly affected by tumor subsites. Another limitation was related to the localization of ROI, which would affect the consistent acquisition of reliable ADC values and TIC. Finally, the semiquantitative dynamic MRI parameters were not included in our study.

\section{CONCLUSION}

The DWI, ADC and the DCE-MRI are emerging tools, which help for discrimination between the recurrent tumor and the post-treatment changes in HNSCC patients. Both DWI and DCE-MRI can be used in conjunction with the conventional MRI technique to confidently diagnose tumor recurrence with increased sensitivity, specificity, and diagnostic accuracy.

Disclosure: The authors report no conflicts of interest in this work.

Source of funding: No Funds, sponsorship or financial support to be disclosed.

\section{REFERENCES}

1. Marur S, Forastiere A (2016): Head and Neck Squamous Cell Carcinoma: Update on Epidemiology, Diagnosis, and Treatment. Mayo Clin Proc., 91 (3): 38696

2. Bourhis J, Sire C, Graff P et al. (2012): Concomitant chemo-radiotherapy versus acceleration of radiotherapy with or without concomitant chemotherapy in locally advanced head and neck carcinoma (GORTEC 99-02): an open-label phase 3 randomised trial. Lancet Oncol., 13 (2): 145-53.

3. Beswick D, Gooding W, Johnson J et al. (2012): Temporal patterns of head and neck squamous cell carcinoma recurrence with positron-emission tomography/computed tomography monitoring. Laryngoscope, 122 (7): 1512-17.

4. Grégoire V, Lefebvre J, Licitra L et al. (2010): Squamous cell carcinoma of the head and neck: EHNS-ESMO-ESTRO Clinical Practice Guidelines for diagnosis, treatment and follow-up. Ann Oncol., 21 (5): 184-6.

5. El Beltagi A, Elsotouhy A, Own A et al. (2019): Functional magnetic resonance imaging of head and neck cancer: Performance and potential. Neuroradiol J., 32 (1): $36-52$.

6. Hwang I, Choi S, Kim Y et al. (2013): Differentiation of recurrent tumor and posttreatment changes in head and neck squamous cell carcinoma: application of high b-value diffusion-weighted imaging. AJNR American Journal of Neuroradiology, 34 (12): 2343-8.

7. Acampora A, Manzo G, Fenza G et al. (2016): High bValue Diffusion MRI to Differentiate Recurrent Tumors from Posttreatment Changes in Head and Neck Squamous Cell Carcinoma: A Single Center Prospective Study. https://www.ncbi.nlm.nih.gov/pmc/articles/PMC4914718/

8. Varoquaux A, Rager O, Dulguerov $P$ et al. (2015): Diffusion-weighted and PET/MR imaging after radiation therapy for malignant head and neck tumors. Radiographics, 35: $1502-27$

9. Becker M, Varoquaux A, Combescure C et al. (2018): Local recurrence of squamous cell carcinoma of the head and neck after radio(chemo)therapy: diagnostic performance of FDG-PET/ MRI with diffusion-weighted sequences. Eur Radio., 28: 651-63.

10. Tshering Vogel DW, Zbaeren P et al. (2013): Diffusionweighted MR imaging including bi-exponential fitting for the detection of recurrent or residual tumour after (chemo)radiotherapy for laryngeal and hypopharyngeal cancers. Eur Radiol., 23: 562-9.

11. King A, Chow K, Yu K et al. (2016): Head and neck squamous cell carcinoma: diagnostic performance of diffusion-weighted MR imaging for the prediction of treatment response. Radiology, 266 (2): 531-8.

12. Narang J, Jain R, Arbab A et al. (2011): Differentiating treatment-induced necrosis from recurrent/progressive brain tumor using nonmodel-based semiquantitative indices derived from dynamic contrast-enhanced T1weighted MR perfusion. Neuro Oncol., 3 (9): 1037-46.

13. Hermans R (2010): Diffusion-weighted MRI in head and neck cancer. Curr Opin Otolaryngol Head Neck Surg., 18 (2): $72-8$.

14. Ailianou A, Mundada P, De Perrot $T$ et al. (2018): MRI with DWI for the Detection of Posttreatment Head and Neck Squamous Cell Carcinoma: Why Morphologic MRI Criteria Matter. AJNR Am J Neuroradiol., 39 (4): 748-55.

15. Jajodia A, Aggarwal D, Chaturvedi A et al. (2019): Value of diffusion MR imaging in differentiation of 
recurrent head and neck malignancies from post treatment changes. Oral Oncol., 96: 89-96.

16. Desouky S, Aboseif S, Shama $S$ et al. (2015): Role of dynamic contrast enhanced and diffusion weighted MRI in the differentiation between post treatment changes and recurrent laryngeal cancers. Egypt J Radiol Nucl Med., 46 (2): 379-89.

17. Vaid S, Chandorkar A, Atre A et al. (2017): Differentiating recurrent tumours from post-treatment changes in head and neck cancers: does diffusionweighted MRI solve the eternal dilemma? Clin Radiol., 72 (1): 74-83

18. Serour D, Adel K, Osman A (2020): Post-treatment benign changes versus recurrence in non-lymphoid head and neck malignancies: can diffusion-weighted magnetic resonance imaging end up the diagnostic challenge?. Egypt J Radiol Nucl Med., 51: 56-63.

19. Jajodia A, Mahawar V, Chaturvedi A et al. (2019): Role of ADC values in assessing clinical response and identifying residual disease post-chemo radiation in uterine cervix cancer. Indian J Radiol Imaging, 29 (4): 404-11.

20. Choi Y, Lee J, Sung Y et al. (2016): Value of Dynamic Contrast-Enhanced MRI to Detect Local Tumor Recurrence in Primary Head and Neck Cancer Patients. Medicine (Baltimore), 95 (19): e3698.

21. Hameeduddin A, Sahdev A (2015): Diffusion-weighted imaging and dynamic contrast-enhanced MRI in assessing response and recurrent disease in gynaecological malignancies. Cancer Imaging, 15: 3-9. 